\title{
Search for Low Mass WIMP and Axions, Emitted from the Sun
}

\author{
B. M. Ovchinnikov", V. V. Parusov \\ Institute for Nuclear Research, Russian Academy of Sciences, Russia
}

Copyright $(2016$ by authors, all rights reserved. Authors agree that this article remains permanently open access under the terms of the Creative Commons Attribution License 4.0 International License

\begin{abstract}
The chamber for direct detection of WIMP with mass $<10 \mathrm{Gev} / \mathrm{c}^{2}$ and axions, emitted from the Sun was developed. The chamber is filled with gas mixture $\mathrm{H}_{2}+3 \mathrm{ppm}$ TMAE $\left(1,5,10\right.$ bar), or $\mathrm{D}_{2}+3$ ppmTMAE. These gas fillings allow to suppress the electron background. For detecting of events is used the system GEM+pin-anode with coefficient multiplication about $10^{5}$. In experiment it is necessary to search the early or daily modulation effects.
\end{abstract}

Keywords Low Mass WIMP, Metallic GEM+Pin-Anode, $\mathrm{H}_{2}+3$ ppm TMAE Filling, Axion

\section{Introduction}

From astronomical observations it is obvious, that the Dark Matter of the Universe exists. Because the WIMP masses $>10 \mathrm{GeV}$ in laboratory experiments are not discovered, it is necessary to search the WIMP with masses $\leq$ $10 \mathrm{GeV}$. J.Va'vra [1] have supposed, that yearly modulation effect in experiments [2] is explained by low mass WIMP scattering on protons of $\mathrm{H}_{2} \mathrm{O}$ molecules which contamination in detector NaI [2].In work [5] it is shown, that the lightest neutralino is the best WIMP Dark Matter candidates.

\section{Methodology of Search for Low Mass WIMP}

In this work for search the low mass WIMP it is proposed the detection chamber [3] with gas mixture $\mathrm{H}_{2}+3 \mathrm{ppm}$ TMAE filling.

The $\mathrm{H}_{2}$ provides as a result of collision with WIMP the recoil protons with energies of a few $\mathrm{keV}$ (see Table 1). The addition of TMAE with a low ionization potential $(5,36 \mathrm{eV})$ provides the recoil protons detection. As another filling of the chamber is used the mixture deuterium + 3ppm TMAE, because:

(1) the energy of D-recoil is two times more than proton recoil.
(2) the spin of nuclear deuterium is equal 1 (the spin of nuclear hydrogen is equal $1 / 2$ ),

This gives the increase the cross section 3-times as compared with hydrogen [6].

The $\mathrm{H}_{2}$-filling provides the electron background suppression, because the recoil protons in $\mathrm{H}_{2}$-medium have the short track [4], as distinguished from background electrons.

For investigation of the mixtures $\mathrm{H}_{2}+3$ ppm TMAE the chamber with detecting system GEM +pin-anode was constructed (Fig 1.). The Chamber was filled with $\mathrm{H}_{2}+3 \mathrm{ppm}$ TMAE under pressure 1, 5 and 10 bar. The results are shown in Fig 2.

The TMAE-addition stabilizes the proportional discharge in detecting system.

The chamber is placed in low background laboratory in low background shielding for search the yearly or daily modulation effects.

\section{Search the Axions, Emitted from the Sun}

For chamber filling is used $\mathrm{D}_{2}+3 \mathrm{ppm}$ TMAE in this experiment.

Because the energy of solar axion is equal to $\sim 1 \mathrm{keV}$ [7], it transfers the energy to recoil deuterium. This allows to suppress the background.

Table 1. Maximum calculated nuclear recoil energy EkeVnr as a function of WIMP mass for two targets: hydrogen and sodium ( $\mathrm{Na}, \mathrm{Ne})[1]$.

\begin{tabular}{|c|c|c|}
\hline WIMP mass $[\mathrm{GeV} / \mathrm{c} 2]$ & Nucleus & EkeVnr[keV] \\
\hline 0.5 & $\mathrm{H}$ & 1.91 \\
\hline 1.0 & $\mathrm{H}$ & 4.30 \\
\hline 1.5 & $\mathrm{H}$ & 6.20 \\
\hline 2.0 & $\mathrm{H}$ & 7.65 \\
\hline 2.5 & $\mathrm{H}$ & 8.78 \\
\hline 3.0 & $\mathrm{H}$ & 9.68 \\
\hline 0.5 & $\mathrm{Na}$ & 0.19 \\
\hline 1.0 & $\mathrm{Na}$ & 0.73 \\
\hline 1.5 & $\mathrm{Na}$ & 1.57 \\
\hline 4.0 & $\mathrm{Na}$ & 9.07 \\
\hline & \multicolumn{2}{|}{} \\
\hline
\end{tabular}




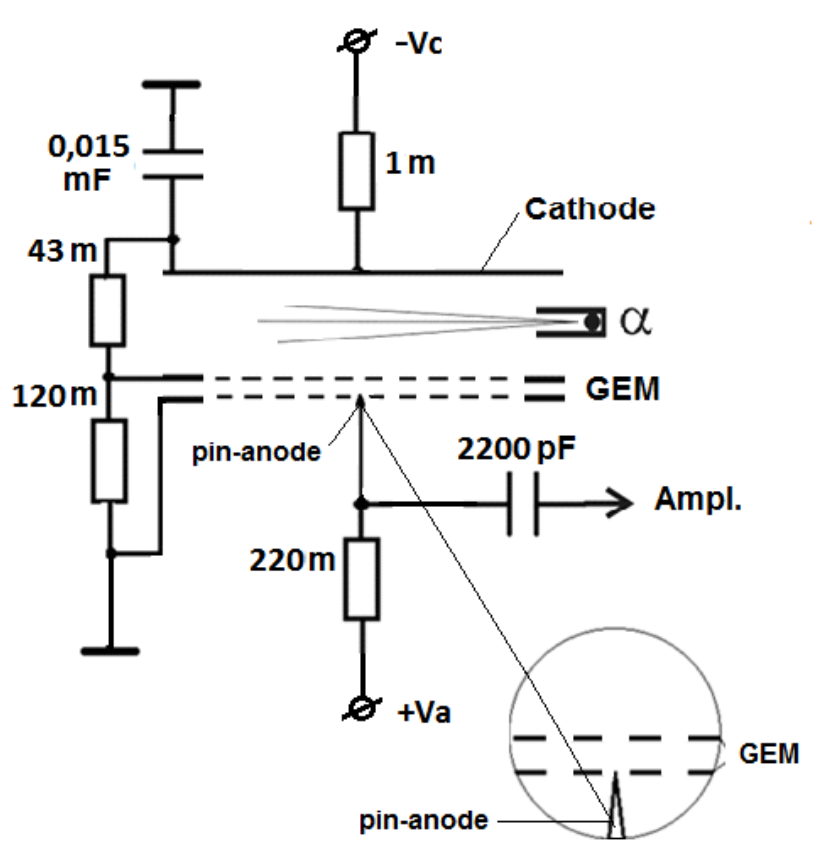

Figure 1. The Chamber with detecting system metallic GEM + pin-anode $[3,8]$ for investigation of the mixture $\mathrm{H}_{2}+3 \mathrm{ppm}$ TMAE. $+\mathrm{Va}=0 \div 3200 \mathrm{~V}$, $-\mathrm{Vc}=580 \mathrm{~V}(5 \mathrm{bar}),-\mathrm{Vc}=860 \mathrm{~V}(10 \mathrm{bar})$.

\section{Results}

The dependence of the electron multiplication factor $\left(\mathrm{K}_{\mathrm{ampl}}\right)$ from potential on the pin-anode $\left(\mathrm{V}_{\text {anode }}\right)$ is shown in Fig. 2.

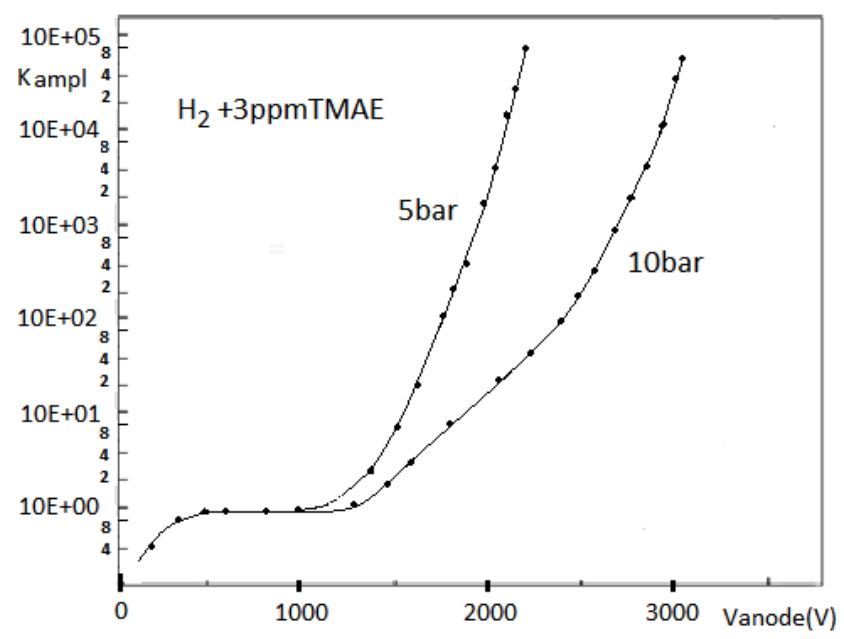

Figure 2. The dependence of $K_{\text {ampl. }}$ from potential on the pin-anode. The TMAE-addition stabilizes the propotional discharge in detecting system. $\left(\mathrm{K}_{\mathrm{ampl}}=\mathrm{Q}_{\mathrm{ampl}} / \mathrm{Q}_{\text {ioniz }}\right.$, where $\mathrm{Q}_{\mathrm{ampl}}-$ the charge detected, $\mathrm{Q}_{\text {ioniz }}-$ the ionization charge).

\section{Discussion}

The hydrogen and deuterium fillings of chamber allow to detect the WIMP with low mass and axion, and to suppres the electron background. The addition in detecting gas the TMAE with low ionization potential increases the sensitivity to low mass WIMP in range $<0,5 \mathrm{GeV} / \mathrm{c}^{2}$. For obtaining the possible minimum WIMP mass with TMAE addition it is necessary to carry out the experiment with low angles neutrons scattering.

\section{Conclusions}

The next steps will include the development of an electronic system for detecting of events in the chamber and construction of the low background shielding in the low background laboratory.

The proposed experiment is most sensitive to low mass WIMP as compared to other experiments. The experiments for search axions, emitted from the Sun, are most simple as compared to other experiments for this aim.

\section{Acknowledgements}

The authors would like to thank Dr. I.I. Tkachev for many useful discussions.

\section{REFERENCES}

[1] J. Va'vra,"A New Possible Way to Explain the DAMA Results", arXiv: 1401. 0698v5, (2014), Physics Letters B 735 (2014)181.

[2] R. Bernabei, P. Belli, F. Cappella, V. Caracciolo, S. Castellano, R. Cerulli, C. J. Dai and A. d'Angelo, "Final model independent result of DAMA/LIBRA-phase1", Eur. Phys. J. C73 (2013) 12, 2648R.

[3] B. M. Ovchinnikov, I.I. Tkachev, V.V. Parusov, "The Metods for Direct Detection of WIMP with Mass $\leq 0,5$ $\mathrm{GeV} / \mathrm{c}^{2}$," Universal Journal of Physics and Application 9(4):173-174, 2015, www.hrpub.org.

[4] R. K. Janev, W. D. Langer, K. Evans et al., "Elementary Processes in Hydrogen-Helium Plasmas", Springer Verlag, Berlin, Heidelberg, New York, London, Paris, Tokyo.

[5] V.V Titkova, V. A. Bednyakov, "Neutralino-nucleon cross section for detection of low-mass WIMP", preprint Dubna E4-2004-131.

[6] V. A. Bednyakov, B.M. Ovchinnikov, V.V. Parusov, "Search for spin-dependent interaction of Dark Matter particles", Preprint INR №1144/2005.

[7] K. Abe, K. Hieda, K. Hiraide et al., "Search for solar axions in XMASS, a large liquid-xenon detector", arXiv: 1212. 6153 (2012), Physics Letters B 721 (2013)46-50.

[8] V. A. Bednyakov, B. M. Ovchinnikov, V.V. Parusov,"Methane ionization chamber to search for spin-dependent dark matter interactions", arXiv: hep-ph/0508052v1, (2005). 